\title{
Higher chlorzoxazone clearance in obese children compared with none-obese peers, an open-label explorative pharmacokinetic study of CYP2E1 activity
}

\author{
Christina Gade ${ }^{1}$, Kim P Dalhoff ${ }^{1}$, Tonny S Petersen ${ }^{1}$, Camilla Schmeltz ${ }^{1,2}$, Elizaveta Chabanova ${ }^{3}$, \\ Hanne R Christensen ${ }^{1}$, Gerd Mikus ${ }^{4}$, Jurgen Burhenne ${ }^{4}$, Jens C Holm², Helle Holst ${ }^{1,2}$ \\ ${ }^{\prime}$ Department of Clinical Pharmacology, Copenhagen University Hospital, Bispebjerg and Frederiksberg, Denmark, \\ ${ }^{2}$ Childrens Obesity Clinic, Department of Pediatrics, Zealand University Hospital, Holbaek, Denmark, ${ }^{3}$ Herlev and \\ Gentofte Hospital, Faculty of Health and Medical Sciences, Copenhagen University Department of Radiology, \\ Denmark, ${ }^{4}$ Department of Clinical Pharmacology and Pharmacoepidemiology, University of Heidelberg, Germany
}

Introduction

In obese adults Cytochrome P450 (CYP) 2E1 activity is induced, probably due to nonalcoholic fatty liver disease (NALFD), diabetes or hyperlipidemia. CYP2E1 has never been investigated in children with obesity. Pathophysiological changes related to obesity may not be as pronounced in children as in adults. Therefore, the pharmacokinetic changes may not be extrapolated into this population.

Aim

To test the in vivo activity of CYP2E1 in obese children vs. nonobese children, aged 11 to 18 years. Secondly, whether the activity of CYP2E1 in these patients is associated with NALFD, diabetes or hyperlipidemia.

Methods

70 children were divided into groups by BMI SDS. All children received $250 \mathrm{mg}$ oral chlorzoxazone (CLZ) as probe for CYP2E1 activity. Thirteen blood samples and twenty-hours urine samples were collected per participant.

Results

Obese children had an increased oral clearance and distribution of CLZ, indicating increased CYP2E1 activity, similar to obese adults. The mean AUC value of CLZ was decreased by $46 \%$ in obese children compared to nonobese children. The mean CLZ Cl was increased twofold in obese children compared to non-obese children, $\mathrm{p}<0.0001$. Diabetic biomarkers were significantly increased in obese children, while fasting blood glucose and Hba1c levels were nonsignificant between groups. Liver fat content was not associated with CLZ Cl.

Conclusion

Oral clearance of CLZ was increased twofold in obese children vs. nonobese children. This indicates an increased CYP2E1 activity of clinical importance, and dose adjustment should be considered for CLZ and probably paracetamol. The latter needs to be further investigated. 